\title{
The Performance of Random and Optimal Scheduling in a Time-Multiplex Switch
}

\author{
CHRISTOPHER ROSE, MEMBER, IEEE, AND MICHAEL G. HLUCHYJ, MEMBER, IEEE
}

\begin{abstract}
In a time-multiplex switching system, the incoming traffic must be scheduled to avoid conflict at the switch output (two or more users converging simultaneously upon a single output). Two scheduling algorithms, random scheduling and optimal scheduling, are explored in this paper. Random scheduling is computationally simple, whereas optimal scheduling is currently very difficult. We have found, using a traffic model appropriate for circuit switched traffic that increases of typically 10 to 15 percent in offered load can be obtained through optimal scheduling (as compared to the much simpler random scheduling algorithm). The improvement is a function of the number of time slots (or circuits) per time-multiplexed frame, and falls to zero for both very small and very large frame sizes. Thus, in many circuit switching applications, providing a computationally expensive optimal schedule may not be warranted. This conclusion has important ramifications for both electronic and emerging photonic switching systems since it reduces the importance of the costly design feature of optimal scheduling.
\end{abstract}

\section{INTRODUCTION}

A TIME-Multiplex switch routes time-multiplexed traffic (or circuits) from its inputs to its outputs. Switching is accomplished by dividing time into "frames" of duration $C \tau$ where $C$ is an integer and $\tau$ is the duration of the elementary time unit, the "time slot." A user requiring one fixed-rate circuit is assigned one free time slot per frame in which to transmit a fixed-length information packet. The switch then routes the packet to the appropriate output during that time slot. This arrangement remains in effect until the user no longer needs the circuit. Since each request for a connection is assumed to arise randomly, and the switch may not send two packets to the same output during the same time slot, some scheduling must be done to avoid conflict at the switch output (two or more users converging simultaneously upon a single output); such conflict would result in the loss of one of the packets.

Optimal scheduling is a means to increase the load which may be offered for a fixed blocking probability and thereby better utilize the capacity of the system. The best utilization would occur were the traffic scheduled at the start of each frame according to user demand. Unfortunately, given a stochastically varying load, the problem of providing such a schedule for each frame is computationally difficult. Therefore, the question arises of whether providing an optimal schedule allows dramatic increases in offered load. Barta and Honig [1] addressed this question for the $N$ user $M$ port case and found little to be gained by computing optimal schedules frame by frame. However, their analysis, primarily for a satellite switching system where $N \gg M$, is not valid for the case $N=M$ as could be expected in a terrestrial switching system wherein each source has a dedicated switch port. This paper addresses the case where $N=M$ for a time-multiplexed switching system in order to examine whether optimal

Paper approved by the Editor for Communication Switching of the IEEE Communications Society. Manuscript received October 6, 1986; revised January $15,1987$.

The authors are with AT\&T Bell Laboratories, Holmdel, NJ 07733.

IEEE Log Number 8715689. scheduling would benefit a land-based communications network.

We have found, using a model appropriate for circuitswitched traffic, that increases of typically 10 to 15 percent in offered load can be obtained through optimal scheduling (as compared to a much simpler "random" scheduling algorithm). This improvement varies with the number of time slots (or circuits) per time-multiplexed frame, and falls to zero for both very small and very large frame sizes. Thus, providing a computationally expensive optimal schedule may not be warranted if the frame size can be varied with relative impunity in a given system. This conclusion has important ramifications for both electronic and emerging photonic switching systems since it reduces the importance of the costly design feature of optimal scheduling. In what follows, the switching system under consideration and the analytic approach to evaluate the system performance are quantitatively described.

\section{Problem Description AND ANALysis}

\section{The Time-Multiplex Switch and a Poisson Traffic Model}

The system under consideration is shown in Fig. $1 . N$ independent sources are connected to separate ports of an $N \times$ $N$ nonblocking switch. Each user is allowed $C$ time slots per frame to transmit information packets to information sinks at the switch output. Each time slot corresponds to one circuit connection. The switch distributes these packets to the appropriate sinks. Each source generates calls according to a Poisson process with rate $\lambda$ and the probability that any given call is destined for sink $j$ is $1 / N$. The call holding time is exponentially distributed with mean $1 / \mu$, thus the normalized average load presented by each source is $\Lambda=\lambda / C \mu{ }^{1}$ The average call holding time $1 / \mu$ and the mean time between arrivals $1 / \lambda$ are assumed to be much greater than the frame length, $C \tau$.

\section{Optimal and Random Scheduling}

During each frame the switch is called upon to supply paths between sources and sinks. Let $t_{i j}$ be the number of time slots requested by source $i$ for transmission to $\operatorname{sink} j$. For $N$ sources and $N$ sinks, the request matrix (traffic matrix) is defined as

$$
T=\left[t_{i j}\right]
$$

where the $t_{i j}$ are nonnegative integers. Given $T$, the system must assign time slots in such a way that two simple constraints are not violated:

1) The number of time slots used by a given source cannot exceed $C$ in a given frame.

2) The number of packets destined for a particular sink cannot exceed $C$ in a given frame.

These conditions correspond to constraints on the row and column sums of $\boldsymbol{T}$. No row or column sum of $\boldsymbol{T}$ may exceed $\boldsymbol{C}$. This condition will be called the "scheduling criterion." It has previously been shown that if $\boldsymbol{T}$ satisfies the scheduling

1 The normalized average Ioad is defined as $\Lambda=(\lambda / C \mu)$ since the maximum number of calls which may be supported by a given port is $C$. 




Fig. 1. An $N \times N$ time multiplex switch.

criterion then it is possible to assign time slots so that no conflict results [2], [3]. Consider adding a single request to a traffic matrix $T$ which satisfies the scheduling criterion. If this new request causes the scheduling criterion to be violated then it must be dropped. If not then the call can be scheduled, although the scheduling may involve rescheduling of calls in progress. A scheduling algorithm is termed optimal if it can provide an assignment for any new request not violating the scheduling criterion. An optimal schedule, however, may be difficult to compute [4], [5]. Thus, alternative suboptimal methods which cause greater packet loss are often used.

The simplest alternative method, random scheduling, is best understood by again considering the addition of a single request to an existing schedule. This request may be granted only if one or more unused time slots on the input and one or more unused time slots on the output coincide in time. If not then the request will be blocked. This concept is illustrated in Fig. 2. Random scheduling is a technique whereby for a given request, such a pair of free time slots is chosen at random from all those available.

\section{A Coupled Markov Chain Model of Input/Output}

Consider the Markov chain of Fig. 3 in which each state represents the number of calls currently active on a given port (input or output). Let $k<C$ calls be active on a given input. The rate at which new call connections are requested by the source is $\lambda$. However, the system will not accept a given call unless the appropriate input to output path is already available (random assignment) or can be made available (optimal assignment). Thus the rate at which calls are accepted by a given input for a given output depends on the number of calls currently active on that input, the number of calls currently active on the output, and for random assignment, the distribution of active calls on the input and output. This acceptance rate, $\lambda_{k}$, will be less than or equal to $\lambda$. The situation is identical for an output port. ${ }^{2}$ Define $\lambda_{k}$ as:

$$
\lambda_{k}=\lambda(1-\operatorname{Pr} \text { [output time slot unavailable } \mid k \text { calls active] })
$$

This idea of modulating the effective arrival rate with the probability of call blockage was first introduced by Lundkvist [6] (see also [7]) in the blocking analysis of a crossbar type switch. The actual form of (2) will, of course, depend upon the system under consideration.

If it is assumed that the sources are independent and that each call generated by source $j$ has probability $1 / N$ of being destined for port $N$, then as $N$ becomes large, the number of calls passing from a given input to a given output becomes negligible relative the total number of calls being handled. This implies that the number of calls currently being handled

2 The system is symmetric in that a request for connection from source $i$ to $\operatorname{sink} j$ may be equally well viewed as a request for connection from sink $j$ to source $i$.

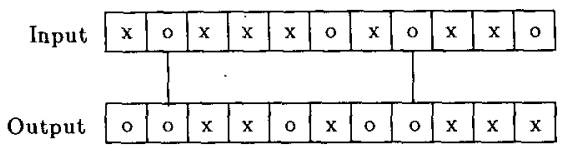

(a)

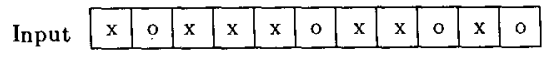

Output \begin{tabular}{|l|l|l|l|l|l|l|l|l|l|l|}
\hline 0 & $\mathrm{x}$ & 0 & $\mathrm{x}$ & $\mathrm{o}$ & $\mathrm{x}$ & $\mathrm{o}$ & $\mathrm{o}$ & $\mathrm{x}$ & $\mathrm{x}$ & $\mathrm{x}$ \\
\hline
\end{tabular}

(b)

Fig. 2. An illustration of coincidence and noncoincidence of free time slots at the output of a time-multiplex switch ( $X=$ occupied time slot, $O=$ free time slot). (a) Two pairs of common time slots. (b) No common time slots.
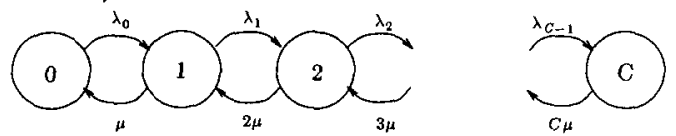

Fig. 3. Discrete-state continuous-time Markov chain model of time-multiplex switch input (or output). Each state corresponds to the number of active calls currently being handled by a given $I / O$ port. The constant $\mu$ is the average call termination rate $\left(1 / \mu\right.$ is the call holding time) and $\lambda_{k}$ is the call acceptance rate from state $k$.

by input $i$ becomes independent of the number of calls being handled by output $j$; the fact that input $i$ is handling $k$ calls gives no information about the number of calls on output $j$. In addition, as $N$ becomes large, the time between requests for connection between a given input and output becomes large compared to the settling time of the Markov chain; each output and input chain approach dynamic independence. The assumption of independence ${ }^{3}$ allows a simple calculation of $\lambda_{k}$.

\section{Derivation of the Call Acceptance Rate, $\lambda_{k}$}

1) Optimal Scheduling: In the case of optimal scheduling, the call request can be granted if the appropriate output has a free time slot. If necessary, all existing connections will be reassigned to different time slots to accommodate the new request. Thus, for a call from source $i$ to sink $j$, blocking will occur only if output $j$ and/or input $i$ is currently handling $C$ calls. Let $I_{\infty}(k)$ be the steady-state probability that $k$ calls are active on a given input and let $\boldsymbol{O}_{\infty}(k)$ be similarly defined for the output to which a connection is sought. Since all the Markov chains are identical, $\boldsymbol{I}_{\infty}(k)=\boldsymbol{O}_{\infty}(k)$. Thus,

$$
\lambda_{k}=\lambda\left(1-I_{\infty}(C)\right)=\lambda\left(1-O_{\infty}(C)\right)
$$

for every input and output chain.

2) Random Scheduling: For random scheduling, the calculation of the conditional probability that the appropriate output slot is available given $k$ calls are active on the curren 1 input is slightly more complex. As illustrated in Fig. 2(b) blocking will occur when there are no free time slots commor to the input and output. Since free slots are chosen at random. assume that any $k$ calls carried by a given port are distributec randomly over the $C$ time slots. Furthermore, since the traffic between any two inputs and outputs is small compared to the total traffic carried by either (large $N$ ), the locations $o$ occupied time slots at the input and output ports will br approximately independent. This assumption allows the fol

${ }^{3}$ The effect of this assumption will be quantitatively evaluated in Appendi. I by a comparison of simulation results and the theoretical results derive herein. It should be noted, however, that the assumption of independence wi. provide an upper bound on the blocking probability. Correlation between a input and output port can only increase the incidence of common free tim slots between the two ports; given that an input slot is unoccupied, correlatio will impose that the corresponding output slot will have a higher probability $c$ being unoccupied as well. 
lowing formulation. Let $i$ be the number of calls on the input and let $\sigma$ be the number of calls on the output. Then,

$$
\operatorname{Pr}[\text { blocked } \mid i]=\sum_{\sigma=0}^{C} O_{\infty}(\sigma) \operatorname{Pr}[\text { blocked } \mid \sigma, i]
$$

The probability that no common free time slot exists given $i$ occupied input slots and $\sigma$ occupied output slots may be found as follows.

Since occupied input and output slots are assumed randomly distributed and independent, it will suffice to consider the input time slot distribution fixed and the output arrangement random. Thus, let the first $i$ time slots on the input be occupied and let the $\sigma$ output slots be randomly distributed (i.e., see Fig. 4). If $i+\sigma<C$ then a common free time slot must exist since the $\sigma$ occupied output slots could not span the $C-i$ free input slots. For $i+\sigma \geq C$ blocking occurs only if $C-i$ of the $\sigma$ occupied output time slots coincide with the $C-i$ unoccupied input time slots. The remaining output slots may be randomly distributed. Thus, out of $\left({ }_{\sigma}^{C}\right)$ possible time slot arrangements, $\left(\begin{array}{c}i \\ C-\sigma\end{array}\right)$ will result in blocking. Therefore,

$$
\operatorname{Pr}[\text { blocked } \mid i, \sigma]=\frac{\left(\begin{array}{c}
i \\
C-\sigma
\end{array}\right)}{\left(\begin{array}{c}
C \\
\sigma
\end{array}\right)}, i+\sigma \geq C \text {. }
$$

The expression of (5a) reduces to

$$
\operatorname{Pr}[\text { blocked } \mid i, \sigma]=\frac{i ! \sigma !}{C !(i+\sigma-C) !}, \quad i+\sigma \geq C .
$$

Substitution of this result into (4) allows $\lambda_{k}$ to be calculated as

$$
\begin{aligned}
\lambda_{k} & =\lambda\left(1-\sum_{\sigma=C-k}^{C} O_{\infty}(\sigma) \frac{k ! \sigma !}{C !(k+\sigma-C) !}\right) \\
& =\lambda\left(1-\sum_{\sigma=C-k}^{C} I_{\infty}(\sigma) \frac{k ! \sigma !}{C !(k+\sigma-C) !}\right)
\end{aligned}
$$

\section{E. Calculating the Steady-State Probability Distribution, $I_{\infty}(k)$}

The equations governing the flow of probability mass in the Markov chain of Figure 3 may be obtained by inspection as,

$$
\dot{I}(k)=\left\{\begin{array}{lr}
-\lambda_{0} I(0)+\mu I(1), & k=0 \\
\lambda_{k-1} I(k-1)-\left(\lambda_{k}+k \mu\right) I(k)+(k+1) \mu I(k+1), \\
\lambda_{C-1} I(C-1)-C \mu I(C), & 1 \leq k \leq C-1 \\
& k=C
\end{array}\right\} .
$$

The chain is ergodic, so for a given set of nonzero $\lambda_{k}, \dot{I}(k)$ will approach zero and a steady-state, $I_{\infty}(k)$ exists. Note, however, that the $\lambda_{k}$ in (7) depend upon the unknown $\boldsymbol{O}_{\infty}(k)=\boldsymbol{I}_{\infty}(k)$ [see (3) and (6)]. This dependence complicates the task of explicitly finding $I_{\infty}(k)$ using equation (7). The method used here is to rewrite the $\lambda_{k}$ in terms of $I(k)$; substitute $\boldsymbol{O}(k)$ for $O_{\infty}(k)$ in equations (3) and (6), and since the input and output Markov chains are identical further substitute $\boldsymbol{I}(k)$ for $\boldsymbol{O}(k)$. Since this substitution renders equation (7) nonlinear, the steady-state distribution must be found numerically.

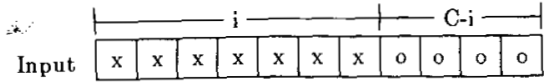

$$
\text { Output } \begin{array}{|l|l|l|l|l|l|l|l|l|l|l|}
\hline 0 & x & 0 & x & 0 & x & 0 & 0 & x & x & x \\
\hline
\end{array}
$$

Fig. 4. Fixed distribution of $i$ occupied time slots on input with random distribution of $\sigma$ time slots on output ( $i=7 \sigma=6$ ).

\section{F. Derivation of Blocking Probabilities}

1) Optimal Scheduling: The probability of blocking for a given load is then

$$
\begin{aligned}
P_{B} & =\sum_{k=0}^{C} I_{\infty}(k) P[\text { blocked } \mid k] \\
& =\sum_{k=0}^{C-1} I_{\infty}(k) O_{\infty}(C)+I_{\infty}(C) \\
& =\left[2-I_{\infty}(C)\right] I_{\infty}(C) .
\end{aligned}
$$

2) Random Scheduling: The probability of blocking for a given load may be calculated by finding the steady-state probability distribution $I_{\infty}(\sigma)$ and using the relation

$$
\begin{aligned}
P_{B} & \left.=\sum_{k=0}^{C} I_{\infty}(k) P \text { [blocked } \mid k\right] \\
& =\sum_{k=0}^{C} I_{\infty}(k) \sum_{\sigma=C-k}^{C} \boldsymbol{O}_{\infty}(\sigma) \frac{k ! \sigma !}{C !(k+\sigma-C) !} \\
& =\sum_{k=0}^{C} I_{\infty}(k) \sum_{\sigma=C \sim k}^{C} I_{\infty}(\sigma) \frac{k ! \sigma !}{C !(k+\sigma-C) !} .
\end{aligned}
$$

\section{Results AND Discussion}

The blocking probabilities resulting from optimal and random scheduling were calculated as functions of percent loading for various frame lengths, $C$. These results are summarized in Fig. 5. It is readily seen that optimal scheduling provides a lower blocking probability $P_{B}$ for a given load. For $C \geq 20$, and reasonable blocking probabilities $\left(<10^{-2}\right)$, optimal scheduling yields a $P_{B}$ approximately one order of magnitude smaller than that supplied by random scheduling. This benefit, however, is misleading since it is possible to achieve a specified $P_{B}$ with random scheduling by decreasing the load a small amount from that at which optimal scheduling achieves the same $P_{B}$. Thus, the difference in maximum achievable load at fixed $P_{B}$ is an important parameter in the comparison of optimal and random scheduling. Define percent improvement as

$$
\text { percent improvement }=\left(\frac{\Lambda^{\text {optimal }}}{\Lambda^{\text {random }}}\right)_{P_{B}=p}-1
$$

where $\Lambda^{\text {optimal }}$ and $\Lambda^{\text {random }}$ are the percent loads at which optimal and random scheduling achieve the required $P_{B}$, respectively.

A plot of percent improvement versus frame length $C$ is provided in Fig. $6 .^{4}$ It is readily seen that no dramatic

\footnotetext{
${ }^{4}$ All the curves must pass through the point $(C$, percent improvement) $=$ $(1,0)$ regardless of the $P_{R}$ criterion; the random assignment and optimal assignment Markov chains are identical for $C=1$. The intercept of each curve with this common point is omitted in Fig. 6 for clarity.
} 


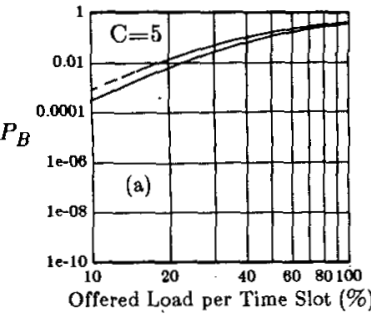

(a)

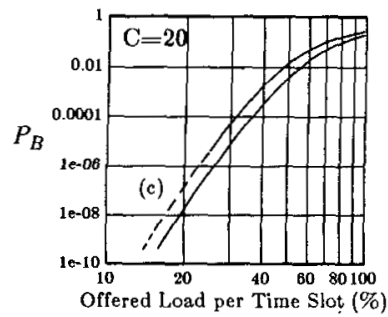

(c)

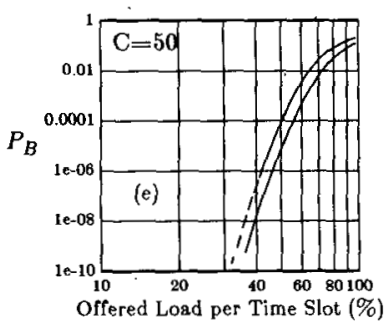

(e)

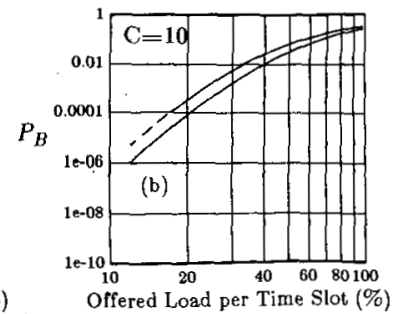

(b)

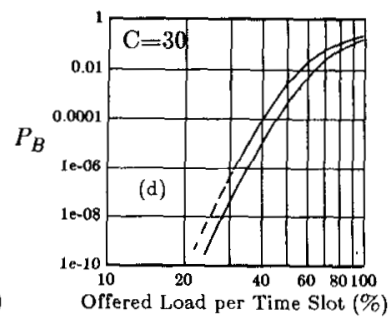

(d)



(f)
Fig. 5. Blocking probability versus offered load per time slot for random scheduling and optimal scheduling; dependence on $C$, the number of time slots per frame; $C$ as shown. Dashed lines represent results from random scheduling and solid lines those for optimal scheduling.

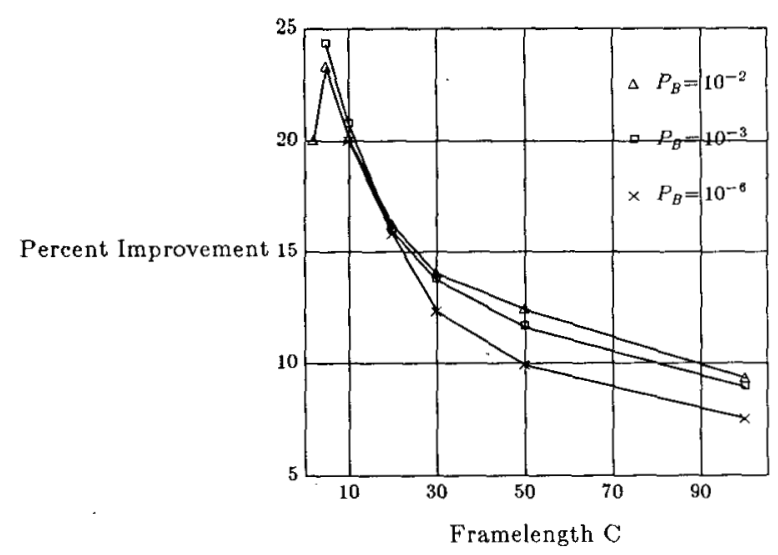

Fig. 6. Percent improvement versus framelength $C$ for fixed $P_{B}$. Triangle$P_{B}=10^{-2}$. Square- $P_{B}=10^{-3} \cdot X-P_{B}=10^{-6}$.

improvements in load-bearing capabilities are attainable using optimal scheduling over random scheduling. The largest improvements are $\approx 20$ percent $(C \leq 10)$. It should be noted, however, that a frame size of $C \leq 10$ produces poor switch performance (maximum load $\approx 40$ percent at $P_{B}=10^{-2}$ ). This poor performance results from the fact that for smaller $C$ there are fewer opportunities for the occurrence of common free time slots between a given input and output. A quantitative illustration how maximum offered load at fixed $\boldsymbol{P}_{B}$ varies

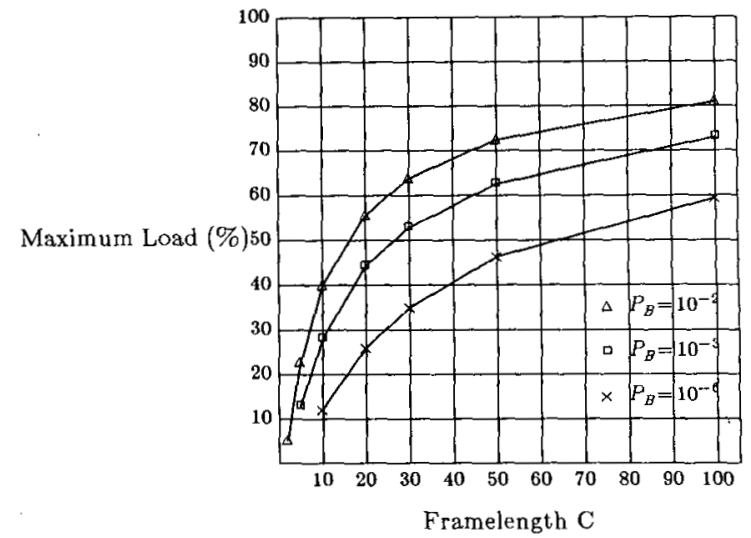

Fig. 7. Maximum load which satisfies $P_{B}=p$ as a function of framelength, $C$ (optimal scheduling). Triangle- $p=10^{-2}$. Square- $p=10^{-3} \cdot X-p=10^{-6}$.

with $C$ is presented in Fig. 7. For more palatable maximum loads ( $>50$ percent) $C$ must be larger than 20 . In this regime of $C$, however, percent improvement lies below 15 percent. Thus, given the complexity of finding an optimal schedule, a sacrifice of $\approx 15$ percent in maximum load may be completely acceptable.

It is also noteworthy that for either scheduling scheme at constant load of less than 100 percent, $P_{B} \rightarrow 0$ as $C$ is increased; even for random scheduling the blocking probability as a function of load will approach a step function $\left(P_{B}=1\right.$ for $\Lambda>1$, zero otherwise). This trend may be seen in Figs. 5 and 6 . Thus, the percentage improvement must approach zero as $C \rightarrow \infty$. Therefore, a given $P_{B}$ could be met with random scheduling by increasing $C$, thereby achieving greater trunking efficiency, and the improvement using optimal scheduling would be minimal. Of course, the bandwidth required by each port and the number of fixed-rate circuits which could be handled would be commensurately larger.

\section{CONCLUSIONS}

For a time-multiplexed switch handling circuit-switched traffic subject to a fixed blocking probability criterion, an increase in the permissible offered load of typically 10 to 15 percent can be achieved by performing optimal scheduling rather than random scheduling of the offered traffic. The derivation of an optimal schedule, however, is a difficult task using current computational techniques. To circumvent this problem and still achieve almost optimal performance, the number of time slots per frame could be increased and a much simpler random schedule employed.

\section{APPENDIX I}

\section{Comparison of Theoretical and Simulation Results}

To test the validity of the independence assumption made in Section II-C, the time-multiplex switch structure was simulated for $C=30$ and various numbers of input ports (users), $N$. For the case of random scheduling, blocking probability as a function of offered load was calculated. The results are compared to the blocking probability versus loading curve for random scheduling found by analysis for $C=30$ (Fig. 8). For $N \geq 20$ the analytic and simulated results are virtually identical. This fact suggests that independence of the input and output Markov chains is a reasonable analytic assumption.

\section{ACKNOWLEDGMENT}

We would like to thank A. S. Acampora for his detailed and thoughtful comments on the manuscript. 


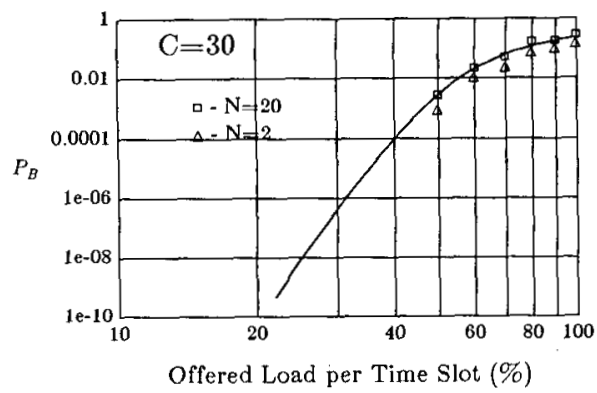

Fig. 8. Blocking probability versus load. Analytic results for random scheduling (solid line) compared to simulation results for two values of $N$ the number of users ( $N$ as shown in legend). Error bars are smaller than the symbol size and are therefore not shown.

\section{REFERENCES}

[1] S. M. Barta and M. L. Honig, "Analysis of a demand assignment TDMA blocking system," AT\&T Bell Lab. Tech. J., vol. 63, no. 1 . pp. 89-114, 1984.

[2] A. S. Acampora and B. R. Davis, "Efficient utilization of satellite transponders via time-division multibeam scanning," Bell Syst. Tech. $J .$, vol. 57 , no. 8 , pp. $2901-2914,1978$.

[3] M. Hall, Jr., Combinatorial Theory, 2nd Ed. New York: Wiley, 1986 , ch. 5.

[4] T. Inukai, "An efficient SS/TDMA time slot assignment algorithm," IEEE Trans. Commun., vol. 27, pp. 1449-1455, 1979.

[5] I. Gopal, D. Coppersmith, and C. K. Wong, "Minimizing packet waiting time in a multibeam satellite system," IEEE Trans. Commun., vol. 30, pp. 305-316, 1982.

[6] K. Lundkvist, "Method of computing the grade of service in a selection stage composed of primary and secondary switches," Ericsson Rev., no. 1 , pp. $11-17,1948$

[7] R. Syski, Introduction to Congestion Theory in Telephone Systems. London: Oliver and Boyd, 1960, ch. 8.



Christopher Rose (S'76-M'86) was born on January 9, 1957 in New York City. He received the B.S., M.S., and Ph.D. degrees in 1979, 1981, and 1985 , respectively, all from the Massachusetts Institute of Technology, Cambridge:

Dr. Rose is currently with AT\&T Bell Laboratories, Holmdel, NJ as a member of the Technical Staff in research. His current technical interests include photonic switching systems, neuromorphic networks, automated character recognition and transmission, as well as the general areas of stochastic, communication, and biophysical systems.



Michael G. Hluchyj (S'75-S'78-S'80-M'82) was born in Erie, PA on October 23, 1954. He received the B.S.E.E. degree in 1976 from the University of Massachusetts, Amherst, and the S.M., E.E. and $\mathrm{Ph} . \mathrm{D}$. degrees in electrical engineering from the Massachusetts Institute of Technology, Cambridge, in 1978, 1978, and 1981, respectively.

From 1977 to 1981 he was a Research Assistant in the Data Communication Networks Group at the M.I.T. Laboratory for Information and Decision Systems where he investigated fundamental problems in packet radio networks and multiple-access communications. In 1981 he joined the technical staff at Bell Laboratories, Holmdel, NJ, where he worked on the architectural design and performance analysis of local area networks. In 1983 he moved with his department to AT\&T Information Systems and continued to work on local area networks. In 1984 he transferred to the Department of Network Systems Research, AT\&T Bell Laboratories, performing fundamental and applied research in the area of high-performance, integrated communication networks for voice, data, image, and video. His current research interests include wide-band circuit and packet switching architectures and multiuser lightwave networks.

Dr. Hluchyj is a member of the IEEE Communications Society and is a member of the Technical Editorial Board for IEEE NETWORK MAGAZINE. 